\title{
CONSTRUCTIONS WITH NEGATIVE PREFIX \\ dT IN THE COPTIC LANGUAGE
}

\author{
By \\ Shaimaa ${ }^{\mathrm{c}}$ Abdelsttar Ahmed \\ Lecturer at Faculty al-Alsun /Luxor University
}

\begin{abstract}
The present paper handles the compound noun, the negative, and the use of the formative $\mathbf{A T}-$, which is used for nouns and verbs since many abstract nouns comprise the prefix at-. Finally, all the abstract nouns negated by $\mathbf{\boldsymbol { A }} \mathbf{T}$ are appended to. In Coptic, there are few true adjectives because the old form of the language is rich in adjective-verbs. Generally speaking, the adjective is expressed in Coptic by means of a relative clause or by substantives linked together by the genitival adjective $\mathbf{N}$-. Less frequently, the noun and its qualifying substantive are in direct opposition. This adjective follows its noun in masculine and feminine forms. In this article, an analysis is made of the use of the negative prefix $\mathbf{a} \mathbf{T}$ - in Coptic. The examples of its use are divided into two groups: - The negative prefix a $\mathbf{x}$ - with verbs; - The negative prefix $\mathbf{A T}$ with nouns. The meaning of some frequently occurring verbal and nominal roots modified by $\mathbf{d} \mathbf{T}-$ is explicated and their etymological (Ancient Egyptian and Demotic) predecessors are provided. Syntactically, the compounds with $\mathbf{d} \mathbf{T}$ - are abstract nouns that function predominantly as equivalents of adjectives, as Coptic has only a few adjectives in the strict sense. Consequently, the $\mathbf{x} \mathbf{T}$ - compounds have adjectival syntactic functions: they modify the head noun in the nominal phrase or function as the nominal predicate in nominal sentences. They can also function as nouns, for example as the subject of the sentence. An overview of the syntactic functions of the $\mathbf{A} \mathbf{T}$ - compounds examined is presented in the conclusion. In the Appendices, alphabetic overviews are provided of respectively the deverbal and denominal compounds with $\mathbf{\lambda} \mathbf{T}$ - mentioned in Crum's Coptic Dictionary. It becomes evident that the deverbal compounds by far outnumber the denominal ones».
\end{abstract}

KEYWORDS: Coptic , negation, prefix , verb , noun, adjective, Late Egyptian , Demotic. 


\section{INTRODUCTION}

The negative prefix $\mathbf{d} \mathbf{T}$ - can change a word's meaning into its antonym, which forms, with nouns and verbs, a negative adjective that means «not, without, un-, in-, -less» ${ }^{1}$.

The prefix $\mathbf{d} \mathbf{T}^{2}, \mathbf{d} \mathbf{T} \boldsymbol{\epsilon}$ comes before double consonants (rarely) 3 , $\mathbf{d \boldsymbol { \theta } ^ { 4 }}$ before the "seven consonants"(B), גeIT $(M)$ derived from hieroglyphic 11 iwtj "which is not"5 and in demotic ¿ $\operatorname{iwt}(j)$ "without"6.

Adjectives are expressed in three ways. The first one is the adjective followed by the noun directly. The noun is then generally in the construct form. There are comparatively few true adjectives in Coptic, e.g., шнм $\rightarrow$ little - young and птоо шнм «the little mountain». The second one utilizes the genitive n- placed between the noun and the adjective. In this form, the noun may either precede or follow its adjective, e.g.,

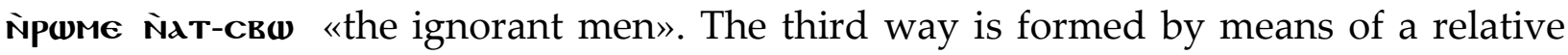
clause used $\mathbf{\epsilon T}$ relative adjective, e.g., пшдхє $\mathbf{\epsilon \mathbf { T }} 2$ «the written word» ${ }^{7}$.

The paper aims to compile an inventory of Coptic lexical units containing the prefix $\mathbf{d T}$ - and classify the examples quoted according to their syntactic functions.

The examples quoted are excerpted directly from Coptic texts (primary sources) and dictionaries (secondary sources).

\section{The negative prefix $\mathbf{x}$ with verbs.}

When the complement is a verb, it can take an object. It is worth noting that when used in a passive sense, the transitive verb must take an object as a pronominal object. It can also join compound verbs to make one negative word.

- The verb $\mathrm{NaY}^{8}=$ look

In late Egyptian $n w$, in Demotic $n w 2 n<10$

aT-NaY (S), do-NaY $(B)+\mathbf{E} / \mathbf{E P O} "$ means unseen, unseeing ${ }^{11}$, invisible (lit. without seeing it).

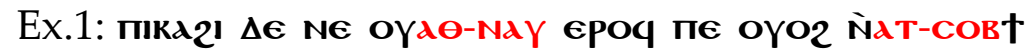

The earth was invisible and empty. $\left(\mathrm{Ge} 1_{2} B\right)$

- The verb MoY $^{12}=$ die

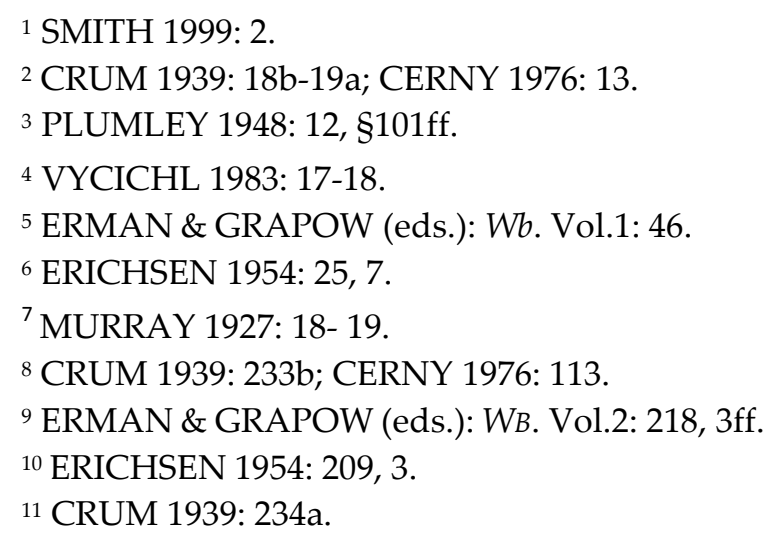


In late Egyptian $m(w) t$

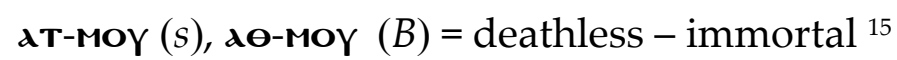

Ex.2: ПАІкגІосүNH Гар оүגт-MOү те.

For uprightness is immortal. (Sa1 $\left.{ }_{15} S\right)$

- The verb Moowe $=$ walk $-\mathrm{go}^{16}$

In late Egyptian $m s^{r}$

aт-moowe $(S)$, גe-moowe $(B)=$ Pathless $^{19}$

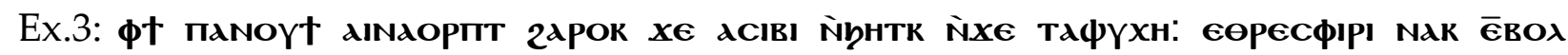

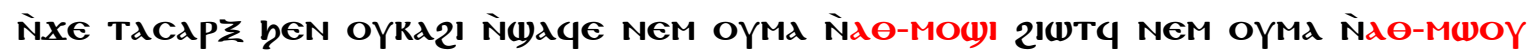

O God, my God, to you, I create because my soul thirsts for you, so that my body may flourish for you in a wasteland, a pathless place, and a place without water. (Ps 62 $B$ )

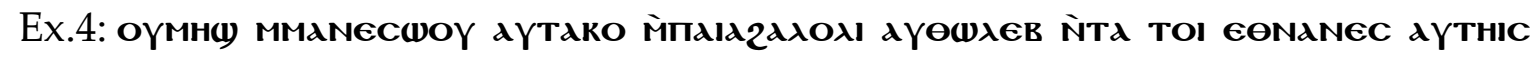

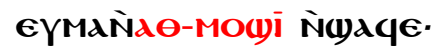

Many shepherds have corrupted my vineyard. They have trampled my portion. They made my inheritance desolate wilderness. (Jer 1210 B)

- The verb ces $(S), \mathbf{c l}(S B)^{20}=$ be filled - satisfied

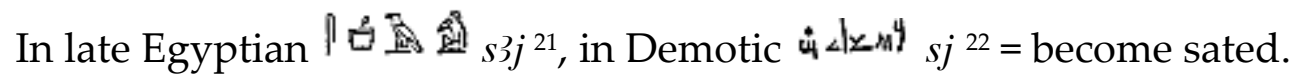

$\mathbf{a} \mathbf{T}-\mathbf{c e l}(S), \mathbf{d T}-\mathbf{c l}(B)^{23}$ (adj.) = insatiate.

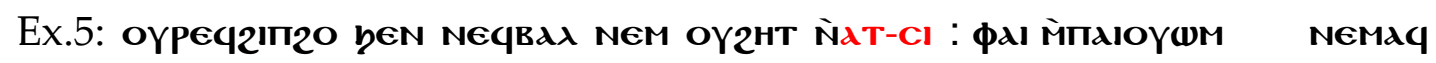

Whoever has haughty eyes and a proud heart, I will not tolerate him. (Ps 1005 B)

- The verb c⿻rm $=$ hear ${ }^{24}$

In late Egyptian $s \underline{d m} 25$, in Demotic stm $(s d m)$ r 26

aT-cotm = inaudible, disobedient ${ }^{27}$

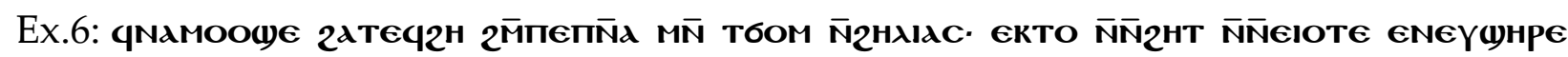

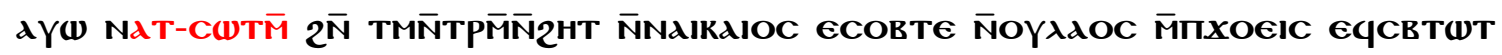

\footnotetext{
${ }^{12}$ CRUM 1939: 159a; CERNY 1976: 79.

${ }^{13}$ ERMAN \& GRAPOW (eds.): $W b$. Vol.2: 165, 8ff.

${ }^{14}$ ERICHSEN 1954: 157, 7.

${ }^{15}$ CRUM 1939: 160 a.

${ }^{16}$ CRUM 1939: 203b; CERNY 1976: 96.

17 ERMAN \& GRAPOW (eds.): $W b$. Vol.2:156,5.

18 ERICHSEN 1954: 181, 1.

${ }^{19}$ CRUM 1939: 205b.

${ }^{20}$ CRUM 1939: 316b; CERNY 1976: 145.

${ }^{21}$ ERMAN \& GRAPOW (eds.): Wb. Vol.4: 14, bottom.

22 ERICHSEN 1954: 407, 5.

${ }^{23}$ CRUM 1939: 317a.

${ }^{24}$ CRUM 1939: 363b; CERNY 1976: 165.

${ }^{25}$ ERMAN \& GRAPOW (eds.): $W b$. Vol.4: 384, 4 f.

${ }^{26}$ ERICHSEN 1954: 478, 4.

${ }^{27}$ CRUM 1939: 364b.
} 
He will walk in front of him in the spirit and power of Helias. To turn the hearts of the fathers to their children, and the disobedient (to walk) in the wisdom of the righteous, to prepare a people for the lord prepared.(Lu $\left.1_{17} S\right)$

- The verb $\mathbf{\epsilon} \mathbf{c}=\mathrm{know}$, understand ${ }^{28}$.

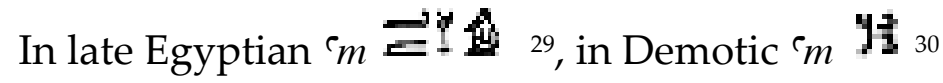

aT-eIme $(S)=$ ignorant, innocent ${ }^{31}$.

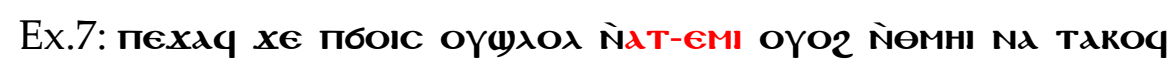

He said: «Lord, would you ignore someone even if he is upright?» (Ge 204 $B$ )

- The verb $\mathbf{\epsilon} \boldsymbol{\omega}, \mathbf{l d},(S B)=$ wash $^{32}$

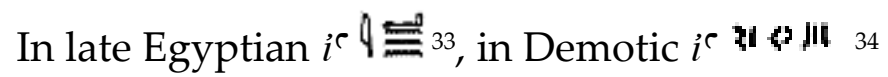

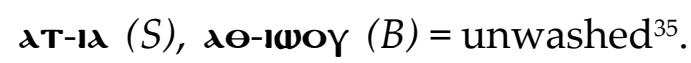

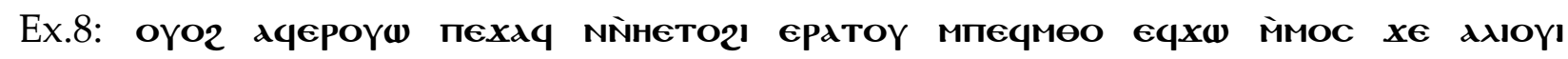

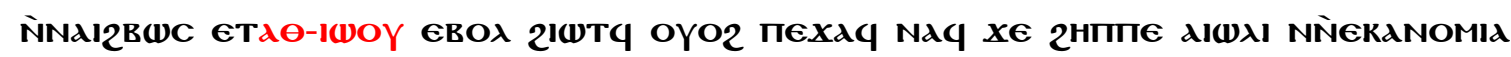

The angel said to those who were standing before him, «Take off his filthy clothes» Then, he said to Joshua, «See, I have taken away your sin, and I will put fine garments on you» $($ Zach 34 B)

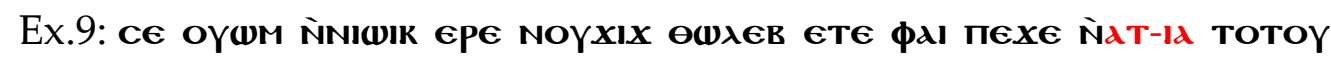

They eat their bread having not washed hands. (MK $72 B$ )

- The verb $\boldsymbol{\kappa \omega}(S), \boldsymbol{x \omega}(B)$ (intransitive verb) = become loose, dissolved ${ }^{36}$

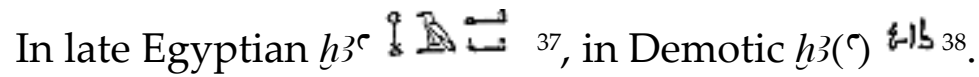

$\boldsymbol{\kappa \boldsymbol { \omega }}(S), \boldsymbol{x} \boldsymbol{\omega}(B)+\mathbf{\epsilon в о \boldsymbol { \lambda }}$ (noun $(\mathrm{m}))=$ freedom, remission, forgiveness.

$\mathbf{\alpha} \mathbf{T}-\boldsymbol{x \boldsymbol { \omega }}$ євох $(B)$ (adj.) = without forgiveness- wrath ${ }^{39}$

- $\mathbf{T} \boldsymbol{\lambda} \boldsymbol{\lambda} \boldsymbol{\sigma} \mathbf{0}(S B)=$ make to cease, heal (causative of $\boldsymbol{\lambda} \mathbf{\lambda} \boldsymbol{\sigma} \boldsymbol{\epsilon})^{40}$

In Demotic $+r / y \frac{1}{3} t j(\Im) \lg { }^{41}=$ cause to stop.

талбо $(S B)($ noun $(\mathrm{m}))=$ healing

дт-тдлбо $(S B)=$ not to be healed. ${ }^{42}$

${ }^{28}$ CRUM 1939: 77b; CERNY 1976: 46-47.

${ }^{29}$ ERMAN \& GRAPOW (eds.): $W b$. Vol.1: 184, 16-20.

${ }^{30}$ ERICHSEN 1954: 60, 6.

${ }^{31}$ CRUM 1939: 78a.

32 CRUM 1939: 75a; CERNY 1976: 45.

${ }^{3}$ ERMAN \& GRAPOW (eds.): $W b$. Vol.1: 39, 2 ff.

${ }^{34}$ ERICHSEN 1954: 48, 6.

${ }^{35}$ CRUM 1939: 75a.

${ }^{36}$ CRUM 1939: 97 a.

${ }^{37}$ ERMAN \& GRAPOW (eds.): $W b$. Vol.3: 227, 3 ff.

38 ERICHSEN 1954: 345, 7.

${ }^{39}$ CRUM 1939: 97b.

${ }^{40}$ CRUM 1939: 411b.

${ }^{41}$ ERICHSEN 1954: 264, 6.

${ }^{42}$ CRUM 1939: 412a. 


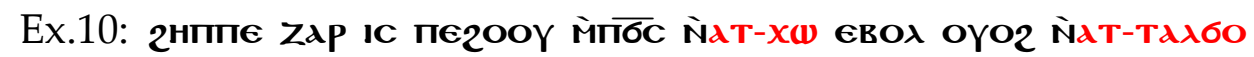

Behold, the day of the Lord comes, cruel, with both wrath and not to be healed. (Is 139

B)

\section{The negative prefix $d T$ - with nouns}

- The noun $\mathbf{B} \boldsymbol{d} \boldsymbol{\lambda}^{43}=$ eye

In late Egyptian $b r \int_{0} 0_{44}$, in Demotic $b l^{s_{2} / 4 / 45}$

$\mathbf{\alpha} \mathbf{T}-\mathbf{B} \boldsymbol{\lambda} \boldsymbol{\lambda}(S)(\operatorname{adj})=$. insatiable - effronte- impudent

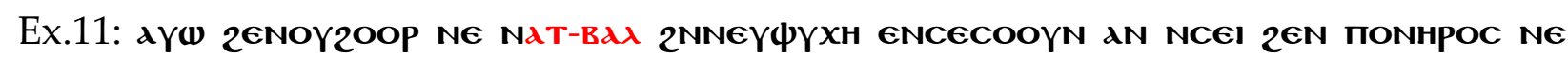
ENCECOOYN AN M̈MNTCABE aYOYAd2OY THPOY NCA NEY2lOOYE חOYA KaTATEqMINE

They are insatiable dogs that know not what it is to be filled, and they are wicked having no understanding at all and having followed their own ways, each according to his will. (Is 5611S)

- The noun $\mathbf{2 H \mathbf { T }}{ }^{46}=$ heart, mind.

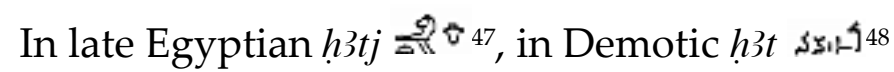

дөнт $(\mathbf{a T}-+\mathbf{2} \mathbf{H T})(S)$ - (adj. $)=$ insensitive, senseless, without mind $^{49}$

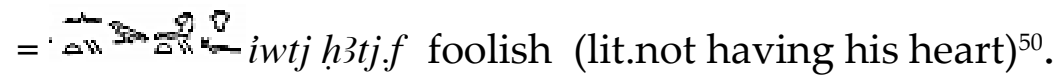

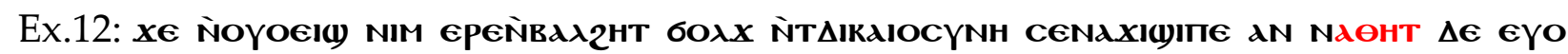

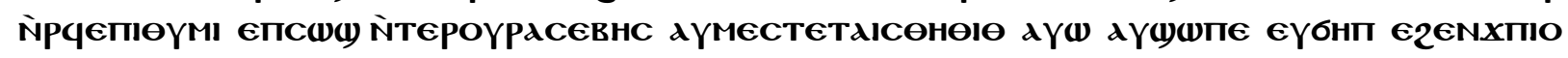
saying, «How long do you fool love ignorance, and mockers take pleasure in mockery, and foolish ones? (Pro $1_{22} S$ )»

- The noun Noвe ${ }^{51}=\sin$

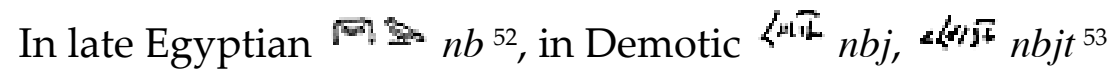
ат-NOвє $(S)$, дө-NOвı $(B)^{54}$ (adj.) = sinless, innocent.

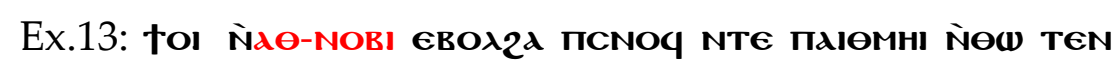
I am innocent of the blood of this just person (MT 27 24 )

- The noun NoYTe ${ }^{55}=\operatorname{god}$.

\footnotetext{
${ }^{43}$ CRUM 1939: 31 b; CERNY 1976: 22.

${ }^{44}$ ERMAN \& GRAPOW (eds.): Wb. Vol.1: 465, 5.

${ }^{45}$ ERICHSEN 1954: 120, I.

${ }^{46}$ CRUM 1939: 714 a; CERNY 1976:.298.

${ }^{47}$ ERMAN \& GRAPOW (eds.): Wb. Vol.3: 26 bottom.

${ }^{48}$ ERICHSEN 1954: 289, 2.

${ }^{49}$ CRUM 1939: 714 b.

${ }^{50}$ ERMAN \& GRAPOW (eds.): Wb. Vol.3: 27,15-16.

${ }^{51}$ CRUM 1939: 222a; CERNY 1976: 106.

52 MOLLER 1842: 543.

${ }^{53}$ ERICHSEN 1954: 214, 6.

54 VYCICHL 1983: 17-18

${ }^{55}$ CRUM 1939: 230b-231a; CERNY 1976: 111. 
In late Egyptian $\Upsilon_{n \underline{t}}{ }^{56}$, in Demotic $\int{ }^{t} \underline{t} r^{57}$

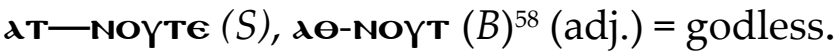

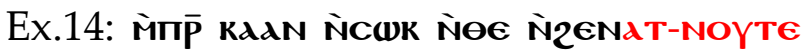

Do not abandon us like the godless (GP 82.b22-23)

- The noun свв $\boldsymbol{\omega}^{59}=$ doctrine, teaching.

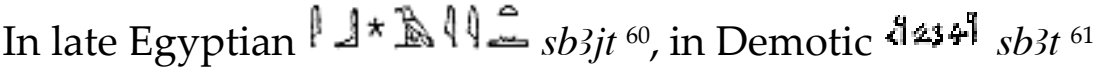

$\mathbf{d T}-\mathbf{C B \boldsymbol { \omega }}(S B)^{62}$ (adj.) = without teaching, ignorance .

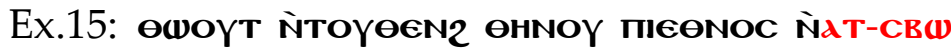

Gather yourselves together, yes, gather together, O ignorant nation. (Zeph $2{ }_{1} B$ )

\section{CONCLUSION}

In conclusion, we could say that there are many prefixes in Coptic that form compound nouns. However, $\mathbf{d T} \mathbf{T}-$, which is added to the noun and the verb to give a negative abstract noun, is a unique formative because it converts the meaning into the antonym or vice versa. It also gives a semantic meaning.

The negative adjective, which comprises the form $\mathbf{a T}-+$ verb or noun follows its noun but is linked together by the genitival $\mathrm{N}^{-}$.

The syntactic functions are:

- compounds as modifiers with the linker: as examples №. 12,14 -15;

- compounds as relatives with the morpheme: like example №. 3, 4, 5, 7, 10;

- compounds as nominal predicates: as examples №. 1, 2, 8, 9, 11;

- compounds as the nominal subject of the sentence: like example №. 6, 13 .

\section{HOW TO CITE}

cAbdelsttar, Sh., « Constructions with negative prefix dT in the coptic language» Journal of the General Union of Arab Archaeologists, vol. 6/2, 2021. Doi: 10.21608/JGUAA2.2021.43523.1037. shaimaaabdelsttar363@gmail.com

\footnotetext{
${ }^{56}$ ERMAN \& GRAPOW (eds.): Wb. II: 358, 1 ff.

${ }^{57}$ ERICHSEN 1954: 232, 6.

58 VYCICHL 1983: 17-18

${ }^{59}$ CRUM 1939: 319b; CERNY 1976: 149.

${ }^{60}$ ERMAN \& GRAPOW (eds.): Wb. IV: 85, $10 f$.

${ }^{61}$ ERICHSEN 1954: 421, 1.

62 VYCICHL 1983: 17-18.
} 


\section{LIST OF ABBREVIATIONS}

$B=$ Bohairic dialect

BG = Berlin Gnostic Papyrus 8502 (cf. Preuss. Akad., Sitz, xxxvi, 839), from photographs

Er = Erichsen, W., Demotisches Glosser, Kopenhagen, 1954

$\mathrm{Ge}=$ Genesis

GP 82.b22-23)

Is $=$ Isaiah

Jer $=$ Jeremiah

Lu $=$ Luka

Mt $=$ Matthew

Mk = Mark

Pro $=$ Proverbs

Ps $=$ Psalms, acc. To the LXX numeration

$S=$ Sahidic dialect

$\mathrm{Sa}=$ Wisdom of Solomon

Wb = Erman, A. \& Grapow, W. (Eds.), Wörterbuch der Ägyptische Sprache, 7 vols und 5 Bde

Belegstellen, Berlin/Leipzig (1926-1963)

Zech $=$ Zechariah

Zeph $=$ Zephaniah

\section{BIBLIOGRAPHY}

CRUM, W., (ed): Coptic Dictionary, Oxford (the Clarendon press), 1939.

CERNY, J., (ed): Coptic Etymological Dictionary, London (Cambridge University Press), 1976.

ERICHSEN, W.: Demotisches Glosser, Kopenhagen (Ejnar Munks Gaard ), 1954.

ERMAN, A.\& GRAPOW, H., (eds.): Wörterbuch der Ägyptischen Sprache I-V, Berlin/Leipzig (J. Hinrichs) (Akademia-Verlag) 1926-1931 [= Wb.].

MOLLER, A.: Edfou de Rochemonteix and E. Chassinat, Le Temple d'Edfou, vol.1, Cairo (Mission Archéologique Française au Caire), 1842.

MURRAY, M.A.: Elementary Coptic(Sahidic) Grammer, London (University College), 1927.

PLUMLEY, J.M.: Introductory Sahidic Coptic Grammar, London (Universty of Cambridg), 1948.

SMITH, R.: A concise Coptic-English lexicon, Atlanta, Georgia, Leipzig (J.C, Hinrichs'sche Buchhandlung), 1899.

VYCICHL, W., (ed): Dictionnaire Etymologique de La langue Copte, Leuven (Peeters Bondgenotenlaan 153), 1983.

WALTERS, C.: An elementary Coptic Grammer, Oxford (University college) , 1972.

WESTENDORF, W.: Koptischen Hand worterbuch, Heidelberg (Carl Winter Universitätsverlag), 1965. 


\section{APPENDICES}

All the English translations are from Crum's Coptic Dictionary

1- dT + verb

$\mathbf{d T}+\mathrm{Verb}$

\section{AT-AMA2TE}

גT-BшTC

AT-KIM

dT-KHN

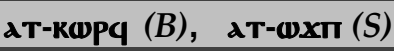

aT-Kat

גT-kTO $(S)$

дт-лшшмє $(S B)$

גT-MKג2 (B)

dT-MOYNK

АT-Mाप्र (SB)

dT-MICe $(S)$

дт-Тмגтe (B)

dT-GMTON (B)

АT-Mєeүє (SB)

גT-Mige (B)

גT-MoүWT (SB)

dT-MoYxo (B)

AT-NA / גe-Nd (SB)

AT-NKOTK $(S B)$

dT-NOGIN $(S)$

dT-N2OYP (B)

AT-NA2TE/AO-NA2TE (SB)

dT-NoYxe (S)

AT-חWWNE (S)

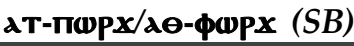

גT-фICE (B)

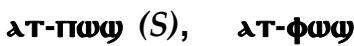

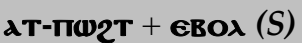

גT-PIKe (SB)

aT-coopr $(S B)$

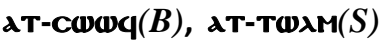

AT-OYWN2, de-OYWN2(B)

dT-OYwP2 $(S)$

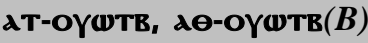

dT-OYXal $(S)$

AT-OYWON $(S)$

גT-

dT-WKM $(B)$

גT-ตா(SB)

АT-WWM $(S B)$

dT- $\operatorname{\omega XN}(S)$

дT-(у)BE $(S)$

\section{Meaning}

unrestrained, uncontrollable

Impugnable

immovable

unending

unceasing

ignorant person

irrevocable

unfading

without pain

unceasing, imperishable

worthless, unworthy

unborn

not agreeing unquiet

without thinking

not attacked, unhurt

intangible

unmixedness

pitiless

sleepless

unshaking

unshakable, immovable unbelieving not cast

immovable, unchangeable undivided, indivisible unmollen

undivided, indivisible not pouring

without turning, unwavering

without knowledge undefiled

unshown, invisible

unallowed, unremitted immutable

unsound, incurable unbroken unalterable without gloom unesteemed unquenchedable, unseaked unceasing unchanging, unaltered 
Shaimaa ${ }^{\mathrm{c} A b d e l s t t a r}$ Ahmed

\begin{tabular}{|c|c|}
\hline גT-लोiBt $(B)$ & unchangeable \\
\hline 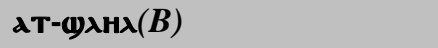 & without prayer \\
\hline גT-ФWNE $(B)$ & without sickness \\
\hline АT-ฒוпє $(B)$ & unashamed \\
\hline 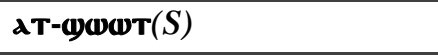 & uncut \\
\hline Ат-๘тортр $(S B)$ & unperturbed \\
\hline גT- $\boldsymbol{D} \lambda \mathrm{x} \in(S B)$ & speechless, unspeakable \\
\hline גT-WOXNe(SB) & being without counsel, ill-considered \\
\hline dT-(y-CAI $(B)$ & unbearable \\
\hline 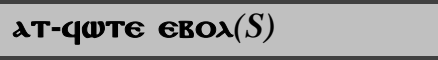 & ineffaceable \\
\hline AT-2WM $(B)$ & untrodden \\
\hline גT-P-2MME(S) & unguided \\
\hline dT-2WN $(S)$ & unapproachable \\
\hline גT-2INHB $(B)$ & without sleep \\
\hline dT-b(WNT $(B)$ & unapproachable \\
\hline dT-2wா(S) & unhidden \\
\hline גT-2POy $(S)$ & without weight \\
\hline dT-2ICe $(S), \quad$ dT-b|cl $(B)$ & unwearied, without difficulty \\
\hline גT-2ITE $(S), \quad$ גT-ИגT $(B)$ & unworn, untormented \\
\hline$d T-x \omega(S)$ & ineffable \\
\hline$\lambda T-x \omega k(S B)$ & without end \\
\hline$\lambda T-x(\epsilon) \mathrm{N} \lambda(S), \lambda T-\sigma \in \mathrm{NO}(B)$ & unquenchable \\
\hline dT-6CWNT $(B)$ & untried, untempted \\
\hline dT-xmog $(S)$ & unbegotten \\
\hline AT- $6 \epsilon p O(B)$ & unlit \\
\hline גT-xpo/бpo(S) & unconquerable \\
\hline dT-XWPT & unimpeded \\
\hline $2 T-x \omega 2 M(S), \quad \lambda T-\sigma \omega_{b \in M}(B)$ & undefiled \\
\hline$\lambda T-x d(\lambda) x \in(S)$ & not rough (hoarse) of voice \\
\hline дT-бомбем $(S)$ & untouchable \\
\hline dT-XINI $(B)$ & not to be found \\
\hline$\lambda T-\sigma(D N T(S), \quad \lambda T-x \omega N T(B)$ & unangered, not to be angered \\
\hline גT-бwாє $(S)$ & not to be taken \\
\hline גT-брш2(B) & without needs \\
\hline Ат-бштп(SB) & without defeat, unconquered \\
\hline
\end{tabular}

\section{$2-\quad d T+$ Noun}

\begin{tabular}{|c|c|}
\hline גT-גITE & Headless \\
\hline AT-APIKE & blameless \\
\hline dT-גYEIN & without cargo \\
\hline$\lambda T-\lambda \sigma N I$ & stainless \\
\hline AT-BGKE & wageless \\
\hline גT-EIWT & fatherless \\
\hline
\end{tabular}


CONSTRUCTIONS WITH NEGATIVE PREFIX גT IN THE COPTIC LANGUAGE

\begin{tabular}{|c|c|}
\hline dT-KळB & without leaven \\
\hline dT-kpoq $(S), \lambda T-x p O q(B)$ & guileless \\
\hline AT-גAdY $(S)$ & without laking any \\
\hline$\lambda T-\lambda 016 \Theta(S B)$ & without cause \\
\hline$\lambda T-M \lambda \operatorname{GIN}(B)$ & without mark \\
\hline dT-MdaY $(S B)$ & without mother \\
\hline גT-Mooy $(S B)$ de-MooY & waterless \\
\hline AT-MaZT $(B)$ & without bowels of compassion \\
\hline AT-NHB $(B)$ & lordless \\
\hline dT-NOMTE $(B$ & without strength \\
\hline dT-NeEq $(S)$ & without sailor \\
\hline dT-NG2, deNG2(SB) & oilless \\
\hline dT-OGIK & without bread \\
\hline 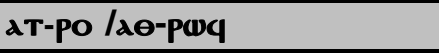 & mouthless, not speaking the language \\
\hline AT-Ka/Xa PW (SB) & without leaving mouth shut \\
\hline dT-PO & doorless \\
\hline גT-PWME $(S B)$ & without man, friendless \\
\hline dT-TPAN $(S)$ & unnamed \\
\hline dT-PoY2є & without evening \\
\hline גT-CMH $(B)$ & voiceless, soundless \\
\hline AT-CMOT & without form \\
\hline AT-CNOG $(S B)$ & bloodless \\
\hline АT-снү $(S B)$ & timeless \\
\hline AT-C्थIME $(B)$ & wifeless \\
\hline dT-OYTaz $(B)$ & fruitless \\
\hline дт-оүмн $(S)$ & without night (sleep) \\
\hline גT-KחE(SB) & numberless \\
\hline dT-(1)גय $(S)$ & toothless \\
\hline dT-bגale $(B)$ & without end \\
\hline AT-2Al $(S B)$ & without a husband \\
\hline AT-21E $(B)$ & rudderless \\
\hline dT-2WB $(F)$ & without work \\
\hline גT-2ג(є)ІвєC $(S)$ & shadowless \\
\hline$\lambda T-P / \epsilon P-200 \lambda \epsilon(B)$ & incomuptible, indestructible \\
\hline$\lambda T-2 \lambda \mathrm{I}(B)$ & without any \\
\hline АT-2MOT(SB) & graceless, thankless \\
\hline AT-2Na $(\lambda) Y$ & state of being without property \\
\hline גT-2גח $(S)$ & without(going to) law, in undertake-ngs to deliver,pay \\
\hline גT-†бגח & not judging \\
\hline dT-bPe $(B)$ & lacking food \\
\hline AT-2גT, $\quad$ AT-бNE2גT(B) & without money, payment \\
\hline גT-2HT(SB) & without mind, senseless \\
\hline גT-2OTE $(S)$ & without fear \\
\hline
\end{tabular}




\section{التراكيب ذات البادئة النافـة فى اللغة القبطية}

\section{شيماء عبدالستار أحمد}

مدرس بكلية الألسن / جامعة الأقصر

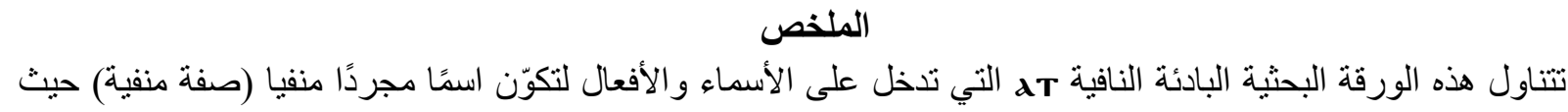

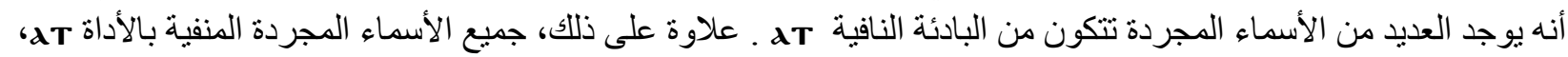

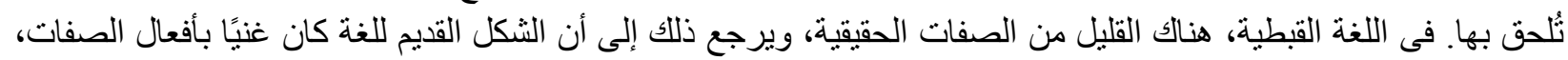

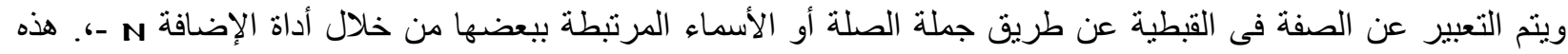

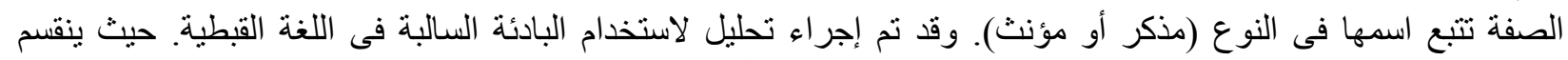

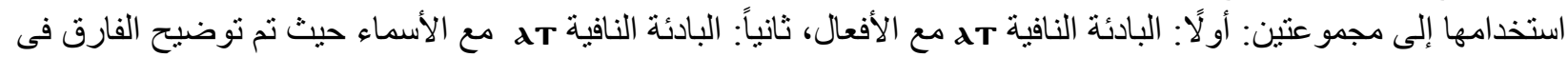

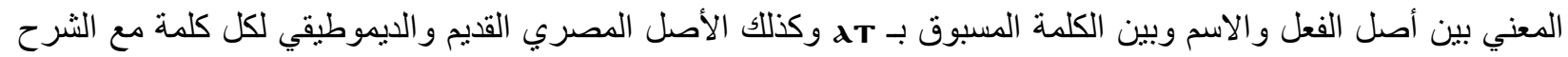

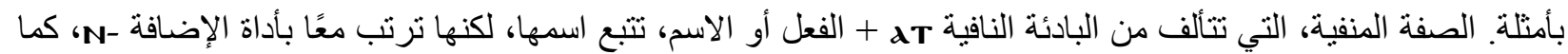

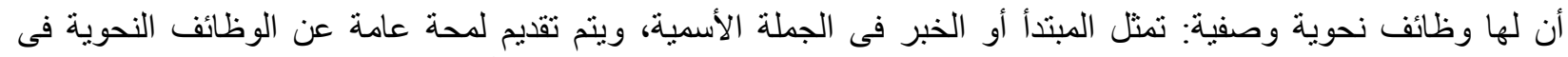

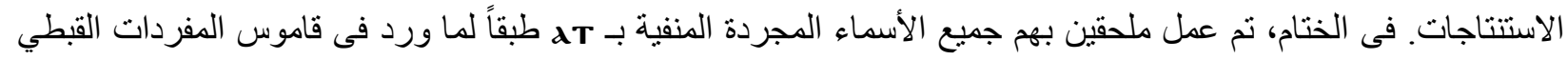

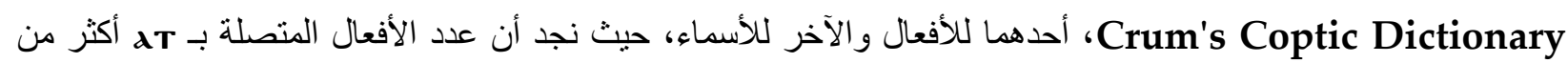
الأسماء المتصلة بـ AT.

الكلمات الدالة: اللغة القبطية، النفى، بادئة، فعل، اسم، صفة، اللغة فى العصر المتأخر ، الديموطيقية. 